XXIII. Kongress der Deutschen Gesellschaft für Philosophie 28. September - 2. Oktober 2014, Münster

Sektion Wissenschaftsphilosophie

\title{
Die multiparadigmatische Struktur der Wissenschaften
}

\author{
Stephan Kornmesser
}

Münstersches Informations- und Archivsystem multimedialer Inhalte (MIAMI) URN: urn:nbn:de:hbz:6-72319610163 


\title{
Die multiparadigmatische Struktur der Wissenschaften
}

\author{
Stephan Kornmesser \\ Sektion Wissenschaftsphilosophie \\ Dienstag, 30. September 2014
}

\section{Einleitung ${ }^{1}$}

Mit Thomas S. Kuhns (1976 [1962]) The Structure of Scientific Revolutions wurde die historische Wende in der Wissenschaftstheorie eingeleitet. Seine deskriptive und diachronische Analyse der Wissenschaften führte zu dem folgenden, wohlbekannten zyklischen Entwicklungsschema: Ist von der wissenschaftlichen Gemeinschaft einer wissenschaftlichen Disziplin ein bestimmtes Paradigma akzeptiert, so konstituiert dieses Paradigma eine normalwissenschaftliche Entwicklung. Das Paradigma beinhaltet forschungsleitende Fragen, methodische Standards, grundlegende theoretische Annahmen sowie Musterlösungen, die auf neue, ähnliche wissenschaftliche Problemstellungen übertragen werden können. Sollten innerhalb der normalen Wissenschaft Anomalien auftreten, werden diese in der Regel durch einen Umbau oder eine Erweiterung des Paradigmas eliminiert. Erst wenn vorhandene Anomalien von der wissenschaftlichen Gemeinschaft als zu schwerwiegend aufgefasst werden, gerät das Paradigma in die Krise. In einer Phase der außerordentlichen Wissenschaft können sich neue Positionen in Konkurrenz zu dem bestehenden Paradigma entwickeln. Setzt sich eine dieser Positionen durch, da es die vorhandenen Anomalien besser zu lösen vermag, verdrängt es das alte Paradigma in einer Phase der wissenschaftlichen Revolution und konstituiert eine neue normalwissenschaftliche Phase, wobei das alte und das neue Paradigma inkommensurabel sind.

Im Zusammenhang mit diesem zyklischen Verständnis wissenschaftlicher Entwicklungen hat Kuhn die drei folgenden Thesen aufgestellt (vgl. Kornmesser und Schurz 2014b, S. 12f.), die in der auf Kuhn folgenden Wissenschaftsphilosophie zu weiten Teilen akzeptiert sind und nur vereinzelt und fragmentarisch kritisiert wurden (vgl. z.B. Feyerabend 1970, Laudan 1977):

1. Wenn in einer Disziplin eine Pluralität von Forschungsansätzen vorliegt, dann handelt es sich um eine vorwissenschaftliche Disziplin. Da vor allem die Nicht-Naturwissenschaften durch eine Pluralität von Forschungsansätzen gekennzeichnet sind, handelt es sich bei ihnen um vorwissenschaftliche Disziplinen, die noch nicht das Stadium einer "reifen" Wissenschaft erlangt haben. Nur die Naturwissenschaften sind "reife" Wissenschaften. Diese These nenne ich die These der Vorwissenschaftlichkeit der Nicht-Naturwissenschaften.

2. Hat eine Disziplin die Phase der Vorwissenschaftlichkeit überwunden, so basiert sie in einer bestimmten Entwicklungsphase immer auf genau einem Paradigma, welches genau eine normalwissenschaftliche Entwicklung konstituiert. Reife Wissenschaften sind somit stets monoparadigmatisch. Diese These nenne ich die These der Monoparadigmatizität der Wissenschaften.

3. Da reife Wissenschaften nach den oben genannten Thesen immer monoparadigmatisch sind, besteht Inkommensurabilität immer zwischen zwei aufeinander folgenden Paradigmen und nie zwischen parallel existierenden Paradigmen. Diese These nenne ich die These der Nicht-Parallelität inkommensurabler Paradigmen.

Im Folgenden werde ich versuchen, die drei genannten Thesen anhand eines wissenschaftsgeschichtlichen Beispiels zu widerlegen. Ich werde zeigen, dass auch Disziplinen, die sich durch eine Pluralität von Forschungsansätzen auszeichnen, entgegen der ersten These als reife Wissenschaften klassifiziert werden und sich entgegen der zweiten These in einem Zustand der Multiparadigmatizität befinden können. Hierzu muss vorausgesetzt werden, dass reife Wissenschaften nicht darüber definiert sind,

\footnotetext{
${ }^{1}$ Sektion Wissenschaftsphilosophie, 30. September 2014, XXIII. Kongress der Deutschen Gesellschaft für Philosophie 2014 in Münster.
} 
dass in ihnen jeweils nur eine wissenschaftliche Position akzeptiert wird. Wäre dies der Fall, wäre eine Widerlegung der ersten These aus rein begrifflichen Gründen unmöglich. Vielmehr gehe ich davon aus, dass die Reife einer Wissenschaft nicht über die Anzahl, sondern über die Qualität der in dieser Disziplin akzeptierten Forschungsansätze bestimmt ist. Die Forschungsansätze müssen die Qualität eines Paradigmas besitzen. Kuhn hat nach seiner anfänglich sehr vagen und uneinheitlichen Bestimmung des Paradigmenbegriffs in Kuhn (1976 [1962], vgl. Masterman 1974 [1970]) den Paradigmenbegriff unter der neuen Bezeichnung disziplinäre Matrix in Kuhn (1976 [1962], Postskriptum und 1978 [1974]) expliziert. Ein Kriterium zur Beantwortung der Frage, ob eine wissenschaftliche Position ein Paradigma ist, müsste somit auf dieser kuhnschen Begriffsexplikation beruhen.

Im zweiten Abschnitt werde ich die in Schurz (1998) sowie in Kornmesser und Schurz (2014b) vorgeschlagene Explikation des Paradigmenbegriffs vorstellen, welche die kuhnschen Komponenten einer disziplinären Matrix beinhaltet und für die Analyse koexistierender Paradigmen entsprechend angepasst ist. Auf dieser Grundlage wird es zu einer wissenschaftsgeschichtlichen, empirischen Frage, ob Disziplinen, in denen eine Pluralität von Forschungsansätzen vorliegt, dennoch als reife Wissenschaften klassifiziert werden können: Wenn die in diesen Disziplinen akzeptierten Forschungsansätze die Eigenschaften eines Paradigmas erfüllen, so handelt es sich um reife Wissenschaften.

Im dritten Abschnitt werde ich zwei nicht-naturwissenschaftliche, linguistische Positionen vorstellen und dafür argumentieren, dass sie die Bedingungen für Paradigmen erfüllen. Bei den beiden linguistischen Positionen handelt es sich um die von Noam Chomsky entworfene Generative Grammatik und um die sich seit den 1980er Jahren entwickelnde Konstruktionsgrammatik. Die Linguistik wird in der Folge (zumindest in dem entsprechenden Teilgebiet) als eine reife Wissenschaft klassifiziert.

Im engen Zusammenhang mit der These der Vorwissenschaftlichkeit der Nicht-Naturwissenschaften steht die zweite o.g. These. Wenn in einer Wissenschaft koexistierende Positionen, die annähernd denselben Forschungsfragen nachgehen, als Paradigmen klassifiziert werden, ist dies eine empirische Falsifikation der These der Monoparadigmatizität der Wissenschaften. Ausgehend von den im dritten Abschnitt vorgestellten linguistischen Paradigmen werde ich gegen die These der Monoparadigmatizität und für die These der Multiparadigmatizität argumentieren. Die Generative Grammatik und die Konstruktionsgrammatik werden sich entgegen der kuhnschen Vorstellung über den zyklischen Entwicklungsverlauf von Wissenschaften als koexistierende rivalisierende Paradigmen herausstellen, die bezüglich annähernd derselben Gegenstandsbereiche entgegengesetzte theoretische Erklärungen anbieten und somit zwei dauerhaft koexistierende Normalwissenschaften konstituieren.

Im vierten Abschnitt werde ich entgegen der dritten o.g. kuhnschen These für die Parallelität inkommensurabler Paradigmen argumentieren. Ausgehend von der Multiparadigmatizität von Wissenschaften ist Inkommensurabilität kein Phänomen, das ausschließlich zwischen zwei aufeinander folgenden Paradigmen auftritt, die durch eine wissenschaftliche Revolution einander ablösen. Stattdessen ist Inkommensurabilität ein Bestandteil der normalwissenschaftlichen Praxis. Hierzu werde ich eine kurze Bestimmung des Inkommensurabilitätsbegriffs geben und anschließend zeigen, dass Generative Grammatik und Konstruktionsgrammatik inkommensurable Paradigmen sind, sowie einige Konsequenzen der daraus entstehenden Problematik diskutieren.

Im fünften Abschnitt werde ich andeuten, welche Folgen die Multiparadigmatizität für die wissenschaftliche Praxis besitzen kann. Dabei unterscheide ich zwischen dem Verhältnis der gegenseitigen Ignoranz, einer destruktiven und einer konstruktiven Rivalität sowie einer metaparadigmatischen Rivalität.

\section{Die Explikation des kuhnschen Paradigmenbegriffs}

Um die Koexistenz von Paradigmen untersuchen zu können, verwende ich den von Schurz (1998) sowie von Kornmesser und Schurz (2014b) verwendeten Begriffsrahmen, der eine Weiterentwicklung des Begriffs der disziplinären Matrix darstellt und auf die Analyse koexistierender rivalisierender Paradigmen ausgerichtet ist. Ein Paradigma besteht demnach aus einer theoretischen, einer empirischen, einer methodologischen und einer programmatischen Komponente.

Die theoretische Komponente entspricht in Kuhns Terminologie den symbolischen Verallgemeinerungen und den Modellen einer disziplinären Matrix. Die theoretische Komponente besteht aus einem Theoriekern, der in Gesetzeshypothesen und Modellvorstellungen untergliedert ist, und den Theorieausprägungen. Die Gesetzeshypothesen sind die grundlegenden theoretischen Annahmen eines Para- 
digmas, die innerhalb des Paradigmas nach Kuhn (1978 [1974], S. 419) gegen empirische Falsifikationen vollständig immunisiert werden. Kuhn bezeichnet solche Gesetzeshypothesen als quasianalytische Sätze und verweist für ein Beispiel auf das zweite Newtonsche Axiom, welches nach Kuhn (1976 [1962], S. 91) "für jene, die sich auf die Newtonsche Theorie festgelegt haben, sich ganz wie eine rein logische Aussage verhalte, die keine noch so zahlreichen Beobachtungen widerlegen können." Die Theorieausprägungen stellen Spezialisierungen, d.h. logische Verstärkungen des Theoriekerns dar, die experimentellen Überprüfungen zugänglich gemacht werden können. Da die Theorieausprägungen den Theoriekern implizieren, folgt aus einer Falsifikation der Theorieausprägungen nicht die Falsifikation des Theoriekerns. Die Modellvorstellungen beinhalten einen ontologischen und heuristischen Rahmen für das Paradigma. Als ein Beispiel für Modellvorstellungen können die in Röhl (2014) herausgearbeiteten unterschiedlichen ontologischen Voraussetzungen der Elektrodynamik des 19. Jahrhunderts angegeben werden: Während in den kontinentalen Ansätzen elektrodynamische Phänomene auf Fernwirkungen zwischen geladenen Objekten zurückgeführt wurden, wurden sie in dem angelsächsischen Paradigma durch bestimmte Zustände eines elektromagnetischen Feldes erklärt. Die beiden unterschiedlichen Modellvorstellungen konstituierten unterschiedliche theoretische Komponenten

Die empirische Komponente eines Paradigmas enthält die erfolgreichen empirischen Anwendungen des Paradigmas und insbesondere die exemplarischen Problemlösungen (Musterbeispiele), die als Orientierungspunkt zur Behandlung neuer, ähnlicher Fragestellungen dienen. Eine Rivalität zwischen koexistierenden Paradigmen entsteht in der Regel dann, wenn ausgehend von den paradigmenspezifischen Musterbeispielen die empirischen Anwendungsbereiche ausgeweitet werden und es zu Überschneidungen der Anwendungen beider Paradigmen kommt. Solche Überschneidungen können allerdings auch künstlich herbeigeführt werden, wie Fischer (2014) zeigt. So wurde in Fischer und Makropoulos (2004) ein und dasselbe soziologische Phänomen aus der Perspektive verschiedener soziologischer Paradigmen untersucht, um die Unterschiede und Beziehungen zwischen diesen herausarbeiten zu können.

Die methodologische Komponente eines Paradigmas enthält die methodischen, epistemologischen und normativen Voraussetzungen eines Paradigmas. Ein Standardbeispiel für Unterschiede in den methodischen Voraussetzungen koexistierender Paradigmen bildet die in Teilen der Sozialwissenschaften stattfindende Kontroverse zwischen quantitativen und qualitativen Forschungsmethoden. Gabriel et al. (2014) diskutieren als Beispiel für ein epistemologisches Element eines Paradigmas die Selbstreferentialität autopoietischer Systeme in Luhmanns Systemtheorie. Als unterschiedliche normative Voraussetzungen koexistierender Paradigmen können beispielsweise die verschiedenen paradigmenspezifischen Haltungen bezüglich der Werturteilsfreiheit in der Frühphase der Entwicklung der Politikwissenschaft betrachtet werden (Lemke und Schaal 2014). Die methodologische Komponente deckt sich zu Teilen mit der Komponente der Werte in einer disziplinären Matrix nach Kuhn.

Die programmatische Komponente ist in Kuhns Konzeption einer disziplinären Matrix nicht vorgesehen. Die programmatische Komponente beinhaltet die Erwartungen innerhalb eines Paradigmas, auf welche Anwendungsbereiche das Paradigma zukünftig ausgeweitet werden kann. Diese Anwendungsbereiche bilden den programmatischen Anwendungsbereich des Paradigmas und entsprechen der positiven Heuristik eines Forschungsprogramms nach Lakatos (1974 [1970]). Die Erweiterung der kuhnschen Konzeption um die programmatische Komponente ist insbesondere für die Analyse von koexistierenden rivalisierenden Paradigmen fruchtbar, da es zu dem Programm eines bestimmten Paradigmas $\mathrm{P}^{1}$ gehören kann, die Erklärungsleistungen eines koexistierenden Paradigmas $\mathrm{P}^{2}$ selbst erbringen zu können. Panaiotidi (2014) diskutiert einen entsprechenden Fall innerhalb der Musikpädagogik, in welchem es zu der programmatischen Komponente des Poietischen Paradigmas gehört, die Anwendungen des rivalisierenden Praxialen Paradigmas zukünftig auch in den eigenen empirischen Anwendungsbereich integrieren zu können.

\section{Die Paradigmen der Generativen Grammatik und der Konstruktionsgrammatik ${ }^{2}$}

In diesem Abschnitt werde ich die Paradigmen der Generativen Grammatik (3.1) und der Konstruktionsgrammatik (3.2) charakterisieren, so dass sie einer weiteren Analyse bezüglich ihrer Rivali-

\footnotetext{
${ }^{2}$ Dieser Abschnitt ist dem Artikel Kornmesser (2014, S. 231-246) entnommen.
} 
tät (3.3) und der zwischen ihnen bestehenden Inkommensurabilität (4.) zugänglich sind. Dabei werde ich in der Darstellung der Paradigmen die zentralen theoretischen Annahmen und Modellvorstellungen, methodologischen Voraussetzungen und empirischen Gegenstandsbereiche jeweils direkt den entsprechenden theoretischen, methodologischen und empirischen Komponenten zuordnen.

\subsection{Die Generative Grammatik}

Die Generative Grammatik ist eine der einflussreichsten linguistischen Theorien seit den 1950ern. In der folgenden Darstellung werde ich mich auf die von Noam Chomsky entwickelte Generative Grammatik beziehen. Chomskys Generative Grammatik kein starres paradigmatisches Gebilde, sondern unterliegt seit den 1950ern einer normalwissenschaftlichen Entwicklung im kuhnschen Sinn. Die folgende Abbildung umfasst die prominentesten Entwicklungsschritte dieses Paradigmas.

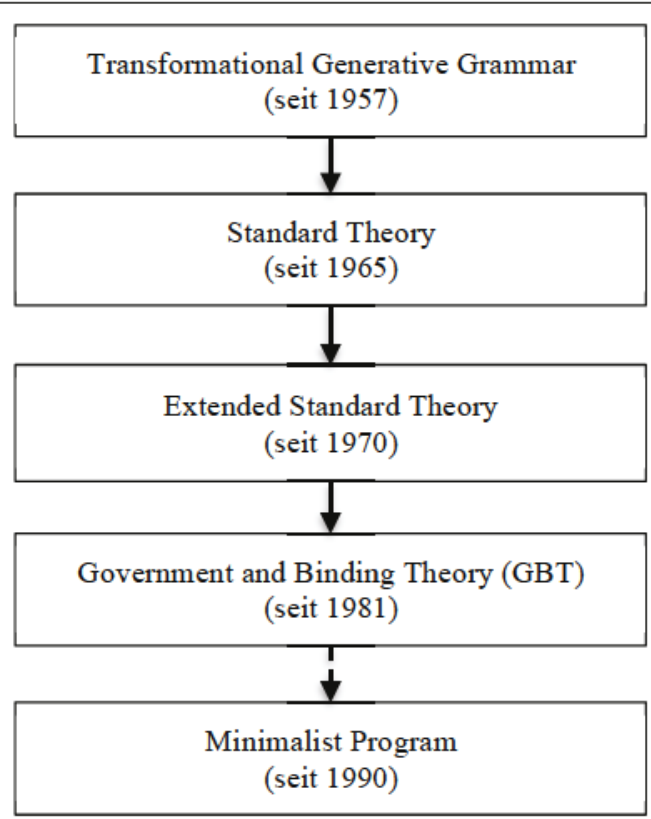

Abbildung 1: Phasen der normalwissenschaftlichen Entwicklung der Generativen Grammatik nach Cook und Newson (2007, S. 4).

Die folgende Darstellung bezieht sich in erster Linie auf die ersten vier Entwicklungsstufen der Generativen Grammatik. Der Übergang zum Minimalistischen Programm ist durch eine gestrichelte Linie symbolisiert, da er sehr umfangreiche Änderungen innerhalb des Paradigmas beinhaltet und hier nicht diskutiert werden kann, inwiefern dieser Übergang noch als eine normalwissenschaftliche Entwicklung zu klassifizieren ist.

Chomskys (1971 [1957]) Syntactic Structures und seine Kritik der behavioristischen Spracherwerbstheorie B. F. Skinners (Chomsky 1959) können als die beiden Ausgangspunkte angesehen werden, die das Programm der Generativen Grammatik initiierten. In Syntactic Structures begründet Chomsky den generativen Aspekt der GG, indem er eine endliche Menge abstrakter (rekursiver) grammatischer Regeln postuliert, mittels derer die potentiell unendliche Menge wohlgeformter Sätze einer Sprache generiert werden können (Generativitätsannahme - Element der theoretischen Komponente). Gleichzeitig kritisiert Chomsky (1959, S. 44) die behavioristische Spracherwerbstheorie von Skinner, indem er zeigt: "there is neither empirical evidence nor any known argument to support any specific claim about relative importance of 'feedback' from the environment [...] in the process of language acquisition“. Chomsky (1959, S. 58) setzt dem behavioristischen Ansatz die Idee einer kognitiven Sprachtheorie entgegen. Nach Chomsky (1965, S. 4) soll das sprachliche Wissen, die Kompetenz, und nicht der tatsächliche Sprachgebrauch, die Performanz, untersucht werden (KompetenzPerformanz-Dichotomie - Element der methodologischen Komponente). Eine grammatische Theorie ist nach Chomsky (1965, S. 24-27) deskriptiv adäquat, wenn sie die Kompetenz eines idealisierten Sprechers einer Sprache beschreibt, und explanativ adäquat, wenn sie den Erwerb der sprachlichen 
Kompetenz erklärt (Spracherwerb als Element der empirischen Komponente). Nach Chomsky muss eine grammatische Theorie sowohl deskriptiv als auch explanativ adäquat sein (Forderung deskriptiver und explanativer Adäquatheit - Element der methodologischen Komponente). Chomsky zeigt allerdings, dass der Erwerb grammatischer Strukturen durch das Kind durch den Sprachinput unterbestimmt ist und nicht als ein induktiver Prozess der Hypothesenbildung und -bestätigung rekonstruiert werden kann (Chomsky 1980b; Chomsky und Fodor 1980). Ein von Chomsky (1975, S. 30-33) gegebenes paradigmatisches Beispiel für die Unterbestimmtheit des sprachlichen Wissens durch den Sprachinput bildet die Strukturabhängigkeit der Transformation deklarativer Sätze in Ja/nein-Fragen. Transformationen der Art

(1) The farmer is mean. $\Rightarrow$ Is the farmer_mean?

stimmen einerseits mit der strukturunabhängigen Hypothese überein, dass bei Transformationen dieser Art das erste verbale Element des Deklarativsatzes an den Anfang des so entstehenden Fragesatzes bewegt wird, und andererseits mit der strukturabhängigen Hypothese, dass das verbale Element nach der ersten Nominalphrase an den Anfang des so entstehenden Fragesatzes bewegt wird. Die beiden Hypothesen unterscheiden sich dadurch, dass nach der ersten ein Satz als eine Abfolge von Wörtern betrachtet wird, in welcher das erste Verb einfach in einer linearen Abfolge bestimmt wird. Die zweite Hypothese setzt voraus, dass Sätze aus Struktureinheiten zusammengesetzt sind, die größer als einzelne Wörter sein können. Diese Struktureinheiten werden Konstituenten genannt. Die Nominalphrase "The farmer" ist eine Konstituente, ebenso wie die Nominalphrase "The farmer who is beating a donkey" in dem folgenden Beispielsatz.

(2) The farmer who is beating a donkey is mean.

Nach Chomsky stimmt der größte Teil des sprachlichen Inputs mit beiden Hypothesen überein, so dass eine induktive Generalisierung der einfacheren strukturunabhängigen Hypothese wahrscheinlich wäre. Trotzdem wird das Kind, so Chomsky, immer die strukturabhängige Hypothese verwenden, die im Gegensatz zur strukturunabhängigen Hypothese für komplexere Konstruktionen die richtigen Ergebnisse liefert, wie die beiden folgenden Anwendungen zeigen:

(3) The farmer who is beating a donkey is mean. $\Rightarrow *$ Is the farmer who _beating a donkey is mean?

(4) The farmer who is beating a donkey is mean. $\Rightarrow$ Is the farmer who is beating a donkey _ mean?

Die von Chomsky gegebenen Beispiele dieser Art können als paradigmatische Beispiele der Generativen Grammatik aufgefasst werden (vgl. Crain und Thornton 2006, S. 1075-1076) und lassen sich auf die allgemeine Form eines Argumentes bringen, das in der Linguistik als das Poverty-of-the-stimulusArgument bekannt geworden ist und wie folgt funktioniert:

1. Kinder lernen in einem gewissen Zeitraum die Grammatik ihrer Sprache.

2. Die Sprachdaten, die die Kinder wahrnehmen, sind nicht ausreichend, um die Grammatik zu lernen.

3. Die Grammatik wird entweder vollständig aus der Erfahrung gelernt oder es gibt angeborenes sprachspezifisches Wissen. ${ }^{3}$

4. Also gibt es angeborenes sprachspezifisches Wissen.

Chomskys Ziel besteht somit darin, das in den ersten beiden Prämissen des Poverty-of-the-stimulusArgumentes beinhaltete, auch als Platons Problem bekannte Spracherwerbsproblem zu lösen, wie ein Kind aus unzureichendem sprachlichen Input die vollständige und richtige Grammatik einer natürlichen Sprache erwerben könne (Chomsky 1986, S. xxv-xxix). Die Lösung von Platons Problem liegt in der Konklusion des Poverty-of-the-stimulus-Argumentes, dass das eine Sprache erwerbende Kind über ein angeborenes sprachliches Wissen verfügt, welches Chomsky als initial state (Chomsky 1980a, S. 87), als language acquisition device (Chomsky 1972, S. 113) oder als universal grammar (Chomsky 1972, S. 27) bezeichnet (Nativismusannahme - Element der theoretischen Komponente). Entspre-

\footnotetext{
${ }^{3}$ Diese Prämisse bleibt in der Regel unexpliziert.
} 
chend der Nativismusannahme verortet Chomsky (1965, S. 47-59, 1966, S. 59-72) die Generative Grammatik innerhalb des philosophischen Programms des Rationalismus, wobei er sich insbesondere auf die Philosophie R. Descartes' bezieht (cartesisch-rationalistische Tradition - Element der methodologischen Komponente). Für Chomsky liegt der entscheidende Unterschied der Generativen Grammatik zu empiristischen Positionen darin, dass die Generative Grammatik sprachspezifische angeborene Ideen postuliert, während empiristische Positionen von allgemeinen kognitiven Mechanismen ausgehen, mittels derer Sprache aus der Erfahrung erworben wird. In der seit 1981 entwickelten GBTVariante der Generativen Grammatik besteht die Universalgrammatik aus Prinzipien, welche universale grammatische Strukturen repräsentieren, und Parametern, die abhängig vom Sprachinput der jeweiligen Einzelsprache während der Erwerbsphase einen variablen Wert annehmen und somit die systematischen Strukturunterschiede zwischen verschiedenen Sprachen erklären (Sprachvergleich Element der empirischen Komponente). Hierbei ist wichtig zu unterscheiden, dass die Parameter selbst nicht erlernt werden, sondern angeboren sind. Lediglich der Wert, den ein Parameter annimmt, wird durch den Sprachinput fixiert. Wenn die Parameter bezüglich der zu erwerbenden Sprache bestimmte Werte angenommen haben, soll die Grammatik dieser Sprache aus der Universalgrammatik ableitbar sein. Diese aus der Universalgrammatik ableitbaren Strukturen einer Sprache nennt Chomsky (1981, S. 7) die Kerngrammatik einer Sprache. Die Kerngrammatik ist von der Peripherie einer Sprache zu unterscheiden, welche die nicht aus der Universalgrammatik ableitbaren Elemente der Sprache enthält (Kern-Peripherie-Dichotomie - Element der methodologischen Komponente). Die Kerngrammatik bzw. die universalgrammatischen Bedingungen der verschiedenen Kerngrammatiken bildet/n den Hauptuntersuchungsgegenstand der Generativen Grammatik. Dabei wird von der Generativen Grammatik nicht ausgeblendet, dass Sprachen eine Peripherie besitzen, aber den für die Generative Grammatik relevanten Gegenstandsbereich bilden die kerngrammatischen Strukturen einer Sprache, da nur diese und nicht die idiosynkratischen Elemente der Peripherie aus grammatischen Regeln bzw. Prinzipien und Parametern erklärt werden können (Chomsky 1981, S. 7-8, 1986, S. 147; Cook und Newson 1996, S. 70-72). Die Kerngrammatik bildet somit den intendierten Anwendungsbereich der Theorie, auf den die theoretischen Annahmen und Modelle der Generativen Grammatik angewendet werden (Ballmer 1976, S. 23).

Chomsky (1980a, S. 38-41 und S. 89, 1981, 2000) postuliert eine modulare kognitive Architektur, nach welcher der menschliche Geist in mehrere funktionsspezifische Module untergliedert ist. Die sprachliche Kompetenz wird als ein Sprachmodul rekonstruiert, welches erstens von anderen Modulen - wie z.B. dem visuellen System - unterschieden und zweitens in weitere Submodule untergliedert ist (Modularitätsannahme - Element der theoretischen Komponente). Für die hier diskutierte Gegenüberstellung zur Konstruktionsgrammatik sind in erster Linie die Komponenten Lexikon und Syntax relevant, die Chomsky (1981, S. 5) als eigenständige Submodule postuliert (Annahme der Syntax-LexikonTrennung - Element der theoretischen Komponente). Das (mentale) Lexikon beinhaltet die idiosynkratischen Elemente einer Sprache, die nicht aus den Prinzipien und Parametern ableitbar sind. Die meisten Elemente des Lexikons sind Wörter, die als Form-Bedeutungs-Paare repräsentiert sind und die für die syntaktische Verarbeitung relevante Informationen enthalten (Chomsky 1986, S. 80-92). Diese syntaktisch relevanten Informationen bestehen zum einen aus der lexikalischen Kategorie des lexikalischen Elementes (Verb, Nomen, Adjektiv, Präposition) sowie aus den Subkategorisierungseigenschaften, d.h. aus der Angabe der Anzahl der geforderten Komplemente. Komplemente sind die neben dem Subjekt von dem Verb geforderten Ergänzungen (beispielsweise benötigt das Verb schlafen im Gegensatz zu dem Verb schlagen kein Komplement). Der lexikalische Eintrag des Verbs (V) to bake mit der Bedeutung [TO BAKE] kann wie folgt dargestellt werden:

(5) to bake [TO BAKE]: V, [-NP]

Die zweite eckige Klammer stellt die Subkategorisierungseigenschaften dar, nach welcher to bake eine Nominalphrase (NP) fordert (z.B. die NP a cake). 
In der GBT-Variante der Generativen Grammatik werden in der Syntax aus den lexikalischen Elementen nach universellen Prinzipien Phrasen und Sätze generiert. Die Prinzipien werden hierbei als einzelne Submodule bzw. Theorien beschrieben (X-Bar-Theorie, Theta-Theorie, Kasustheorie etc.). Die $\mathrm{X}$-Bar-Theorie ist ein fundamentales Prinzip für die Generierung von Phrasen, die in Phrasenstrukturbäumen dargestellt werden können. ${ }^{4}$ In Abbildung 2 ist das Verb bake der Kopf der Verbalphrase (VP) bake a cake. Die Nominalphrase (NP) a cake ist das Komplement des Verbs und wird von diesem regiert (es weist ihm z.B. einen - in der Oberflächenstruktur nicht immer sichtbaren - Kasus zu). Die VP ist die maximale Projektion des Kopfes V. Der Kopf V bildet zusammen mit seinem Komplement



Abbildung 2: Phrasenstrukturelle Darstellung des Satzes "Sally baked a cake" entsprechend der X-Bar-Theorie.

die Zwischenprojektion V` (V-Bar). Zwischenprojektionen können mit nicht vom Verb geforderten Konstituenten (Adjunkten) weitere Zwischenprojektionen bilden. Z.B. entstünde in dem Satz Sally baked a cake in the morning zwischen VP und V' eine weitere Zwischenprojektion V', die sich in die in Abbildung 2 angegebene $\mathrm{V}^{\prime}$ und die Präpositionalphrase (PP) in the morning verzweigte. Der Kopf I der Phrase IP ist im Gegensatz zu den lexikalischen Kategorien V, N, A und P eine funktionale Kategorie, welche die grammatischen Merkmale Tempus und Kongruenz des Verbs repräsentiert. Die Nominalphrase Sally bildet das Subjekt des Satzes und steht in der Spezifikatorposition der IP. Die Dreiecke kennzeichnen, dass die zugehörigen Phrasen nicht weiter analysiert wurden.

Während die X-Bar-Theorie als ein universelles Prinzip postuliert wird, ist die Reihenfolge von Kopf und Komplement parametrisiert. Beispielsweise folgt im Englischen das Komplement seinem Kopf, im Japanischen hingegen folgt der Kopf seinem Komplement.

Systematische Transformationsprozesse - wie z.B. die Transformation von einem Aktiv- in einen Passivsatz oder von einem Aussage- in einen Fragesatz - werden von Chomsky $(1957,1965,1981,1986)$ durch Transformationen von einer Tiefenstruktur (später: D-Structure) in eine Oberflächenstruktur (später: S-Structure) erklärt (Transformationsannahme - Element der theoretischen Komponente). Die grammatischen Strukturen werden entsprechend der X-Bar-Theorie aus Elementen des Lexikons in der Tiefenstruktur generiert und anschließend - beschränkt durch weitere Prinzipien - in die Oberflächenstruktur überführt, von der aus sie phonetisch und semantisch interpretiert werden. Die Beschreibung und Klassifizierung grammatischer Phänomene anhand des dargestellten Instrumentariums ist Bestandteil des Gegenstandsbereiches der Generativen Grammatik (Beschreibung und Klassifizierung grammatischer Phänomene - Element der empirischen Komponente).

\footnotetext{
${ }^{4}$ Aus Umfangsgründen kann die X-Bar-Theorie hier nicht dargestellt werden. Stattdessen wird in Abbildung 2 ein entsprechend der X-Bar-Theorie generierter Phrasenstrukturbaum dargestellt und erläutert, um erstens einen intuitiven Eindruck in die generativistische Satzanalyse zu geben und zweitens anhand einer konstruktionsgrammatischen Analyse im nächsten Abschnitt die Unterschiede beider Positionen zu verdeutlichen.
} 


\subsection{Die Konstruktionsgrammatik}

Unter dem Begriff Konstruktionsgrammatik werde ich im Folgenden eine Familie von Ansätzen zusammenfassen, die zentrale Grundannahmen teilen und sich alle als Abgrenzung zur Generativen Grammatik verstehen. Ich unterscheide vier zentrale Varianten der Konstruktionsgrammatik: die auf den Arbeiten von C. Fillmore et al. (1988) und P. Kay und C. Fillmore (1999) basierende Construction Grammar, die von A. Goldberg $(1995,2006)$ entwickelte und auf G. Lakoff (1987) zurückgehende Cognitive Construction Grammar sowie die Radical Construction Grammar von W. Croft (2001). Des Weiteren werde ich die Cognitive Grammar von R. Langacker (1987) sowie die Arbeiten von M. Tomasello (2003), die in eine konstruktionsgrammatische Rahmentheorie eingebettet sind, in diese Paradigmenfamilie mit einschließen. ${ }^{5}$ In der Konstruktionsgrammatik können bezüglich dieser verschiedenen Varianten unterschiedliche Spezialisierungen in den Bereichen der empirischen Anwendungen ausgemacht werden. Tomasello (2003) verwendet eine konstruktionsgrammatische Rahmentheorie zur Erklärung des Spracherwerbs (Spracherwerb - Element der empirischen Komponente), die Cognitive Grammar von Langacker (1987) und die Cognitive Construction Grammar von Goldberg $(1995,2006)$ analysieren grammatische Phänomene, die sich auf Konstruktionen zurückführen lassen, und schlieBen hieraus auf die kognitive Repräsentation sprachlichen Wissens und die Radical Construction Grammar von Croft (2001) widmet sich in erster Linie sprachvergleichenden Untersuchungen (Sprachvergleich - Element der empirischen Komponente). Die Beschreibung und Klassifizierung grammatischer Phänomene (Element der empirischen Komponente) als Konstruktionen bzw. als Instanziierungen von Konstruktionen ist Bestandteil des empirischen Gegenstandsbereiches aller Varianten der Konstruktionsgrammatik.

Als Ausgangspunkt der Entwicklung der Konstruktionsgrammatik können die Analysen idiosynkratischer Elemente einer Sprache durch Fillmore et al. (1988) und Lakoff (1987, S. 462-585) angesehen werden. Idiomatische Ausdrücke sind dadurch bestimmt, dass ihre Syntax nicht aus einer Menge allgemeiner Regeln abgeleitet werden kann und/oder ihre Bedeutung sich nicht unter Verwendung kompositionaler Regeln aus der Bedeutung ihrer Teilausdrücke und der Art ihrer Zusammensetzung ergibt. Vielmehr müssen diesen idiosynkratischen Elementen eine spezifische Syntax (Form) und Semantik (Bedeutung) direkt zugeordnet werden, so dass sie als eigenständige Form-BedeutungsPaare erscheinen. Typische Beispiele für idiosynkratische Form-Bedeutungs-Paare bilden die folgenden Idiome:

(1) kick the bucket

(2) all of a sudden

Nach Fillmore et al. (1988) gilt für jedes dieser Idiome, dass ihre Semantik oder ihre Syntax nicht aus allgemeinen Regeln abgeleitet werden können und sie somit als eigenständige Einheiten im sprachlichen Wissen eines Sprechers des Englischen repräsentiert sein müssen. In der Generativen Grammatik werden Idiome als Elemente des Lexikons angenommen, da sie wie Wörter gelernt werden müssen und aufgrund ihrer Idiosynkrasie nicht dem Bereich der Kerngrammatik zugeordnet werden können. Dies ist ein gangbarer Weg für diejenigen Idiome, die Fillmore et al. (1988, S. 505) substantive (lexically filled) idioms nennt. Ein substantive idiom wie kick the bucket ist eine feststehende Wendung mit einer feststehenden Bedeutung, die als eine nichtveränderbare Einheit gelernt und im Lexikon repräsentiert werden kann. Ein formal (lexically open) idiom hingegen ist produktiv in dem Sinne, dass es bestimmte Leerstellen enthält, die nach für das Idiom spezifischen Regeln gefüllt werden können, wodurch der Gesamtausdruck eine für das Idiom spezifische Bedeutung erhält. Ein Beispiel für ein formales Idiom ist das Schema

\section{(3) The X-er the Y-er}

Dieses Schema kann durch Einsetzungen in die Variablen X und Y beispielsweise wie folgt instanziiert werden:

\footnotetext{
${ }^{5} \mathrm{Nicht}$ in die Betrachtung mit einbezogen sind die Embodied Construction Grammar, die Sign-Based Construction Grammar und die Fluid Construction Grammar. Für einen Überblick vgl. Ziem und Lasch (2013, S. 57-66).
} 
(3.1) The more you learn, the smarter you get.

(3.2) The faster you run, the harder you fall.

Das Schema (3) ist produktiv und kann somit trotz seiner Idiosynkrasie nicht als geschlossene Einheit in das Lexikon integriert werden. Andererseits kann das Schema (3) wegen seiner Idiosynkrasie in der Generativen Grammatik nicht in den Bereich der Kerngrammatik aufgenommen werden. Somit müsste (3) aus dem Bereich der intendierten Anwendungen der Generativen Grammatik, der sich auf die unter den Begriff Kerngrammatik fallenden Phänomene bezieht, ausgeschlossen werden, obwohl es eine syntaktisch komplexe und produktive Einheit darstellt. ${ }^{6}$

Die Konstruktionsgrammatik beschreitet in ihrem Theorieaufbau einen zur Generativen Grammatik entgegengesetzten Weg: Anstatt idiomspezifische Analysen aus dem Bereich der intendierten Anwendungen auszuschließen, bilden diese in der Konstruktionsgrammatik die Menge der die Theorie motivierenden paradigmatischen Anwendungen. Der von der Idiom-Analyse zur Konstruktionsgrammatik führende Weg kann als aus einer Definition und einer postulierten Verallgemeinerung bestehend beschrieben werden. Die Definition besteht in einer Bestimmung des Begriffs Konstruktion (Construction) als eines Form-Bedeutungs-Paares. Eine erste Definition des Begriffs Konstruktion findet sich in Lakoff $\left(1987\right.$, S. 467): ${ }^{7}$

Each construction will be a form-meaning pair $(F, M)$ where $F$ is a set of conditions on syntactic and phonological form and $\mathrm{M}$ is a set of conditions on meaning and use.

Goldberg (1995, S. 4) verschärft diese Definition um die Bedingungen der Nicht-Kompositionalität und der Nicht-Ableitbarkeit aus anderen Konstruktionen wie folgt

$\mathrm{C}$ ist a construction iff ${ }_{\text {def }} \mathrm{C}$ is a form-meaning pair $\left\langle\mathrm{F}_{i}, \mathrm{~S}_{i}>\right.$ such that some aspect of $\mathrm{F}_{i}$ or some aspect of $S_{i}$ is not strictly predictable from C's component parts or from other previously established constructions.

und schwächt diese Definition in Goldberg (2006, S. 5) in Abhängigkeit von Frequenzeffekten von Konstruktionen wieder ab:

In addition, patterns are stored as constructions even if they are fully predictable as long as they occure with sufficient frequency.

Eine Konstruktion kann somit als eine symbolische Einheit verstanden werden, die aus einer Formund einer Bedeutungskomponente besteht (Langacker 1987, S. 58). In diesem Sinne erfüllen Konstruktionen die klassische Definition eines Zeichens im Sinne de Saussures. Nach dieser Definition ist jedes Idiom eine Konstruktion, da es eine spezifische Form und eine spezifische Bedeutung besitzt.

Die postulierte Verallgemeinerung besteht darin, dass nicht nur Idiome Konstruktionen sind, sondern dass sprachliche Elemente auf allen Ebenen sprachlicher Beschreibung und grammatischer Repräsentation als Konstruktionen aufzufassen sind (Croft 2001, S. 17 und Goldberg 2006, S. 18). Somit werden beispielsweise auch Wörter, Derivations- und Flexionsmorphologie sowie abstrakte Argumentstrukturen als Konstruktionen, also als Form-Bedeutungs-Paare aufgefasst. Für Wörter bzw. Idiome ist es unmittelbar einsichtig, dass sie dem Kriterium, ein Form-Bedeutungs-Paar zu sein, genügen. Syntaktische Kategorien wie die Nominalphrase (NP) oder abstrakte Argumentstrukturen wie die Ditransitivstruktur gelten allerdings in der Tradition der Generativen Grammatik als rein syntaktische Elemente, die keinen Bedeutungsanteil besitzen und somit prinzipiell von den Einheiten des Lexikons zu unterscheiden sind. In der Konstruktionsgrammatik hingegen werden Wörter und grammatische Strukturen als Bestandteile eines Kontinuums aufgefasst, da sie beide als Konstruktionen verstanden werden und somit kein prinzipieller Unterschied zwischen ihnen besteht. Beispielsweise wird die Ditransitivstruktur in der Konstruktionsgrammatik als ein Form-Bedeutungs-Paar aufgefasst, das im Gegensatz

\footnotetext{
${ }^{6}$ Müller (2011, S. 213f.) diskutiert idiomspezifische Regeln, um regelbasierte Erklärungen auch für idiosynkratische Elemente anbieten zu können. Dies sind allerdings typische Fälle von Ad-Hoc-Annahmen, um idiosynkratische Elemente als Anwendungsfälle regelbasierter Ansätze aufrechtzuerhalten.

${ }^{7}$ Variationen dieser Begriffsbestimmung finden sich in Croft (2001, Kap. 1.3.2) und Langacker (1987, S. 57-60, 82 und 409).
} 
zu Wörtern und Idiomen allerdings sehr schematisch ist. Die schematische Form besteht in der Struktur [Subj Verb $\mathrm{Obj}_{1} \mathrm{Obj}_{2}$ ], die beispielsweise wie folgt instanziiert werden kann:

\section{(4) Sally baked her sister a cake. ${ }^{8}$}

Die schematische prototypische Bedeutung, die durch die Konstruktion ausgedrückt wird, besteht in dem intendierten Transfer des $\mathrm{Obj}_{2}$ vom Subj zum $\mathrm{Obj}_{1}$. Da diese Bedeutung des intendierten Transfers in (4) kein Bestandteil des Verbs "to bake" oder anderer lexikalischer Elemente aus (4) ist, muss dieser Bedeutungsaspekt durch die Konstruktion induziert sein (Goldberg 1995, S. 141). Die konstruktionsgrammatische Darstellung der Ditransitivkonstruktion ist in vereinfachter Form in Abbildung 3 wiedergegeben. Die Konstruktion besteht aus einer Formseite (Syn), die aus einem Verb (V), einem Subjekt (Subj), einem Objekt ${ }_{1}\left(\mathrm{Obj}_{1}\right)$ und einem Objekt ${ }_{2}\left(\mathrm{Obj}_{2}\right)$ besteht, sowie einer Bedeutungsseite (Sem), welche die Bedeutung CAUSE-RECEIVE <agt, rec, pat> beinhaltet: Ein agent (agt) intendiert zu verursachen, dass ein recipient (rec) ein patient (pat) empfängt. PRED ist eine Variable, die durch ein bestimmtes Verb instanziiert wird, welches in die Konstruktion eingesetzt wird. Die gestrichelte Linie von rec bedeutet, dass diese semantische Rolle nicht obligatorisch durch das Verb vorgegeben werden muss, sondern durch die Konstruktion selbst induziert werden kann. Wird beispielsweise im obigen Satz (4) baked durch gave ersetzt, so ist die semantische Rolle rec durch das Verb obligatorisch gefordert. Das Verb baked in (4) hingegen fordert nicht obligatorisch ein $\mathrm{Obj}_{1}$ und die semantische Rolle rec wird im Fall von (4) durch die Konstruktion selbst beigesteuert.

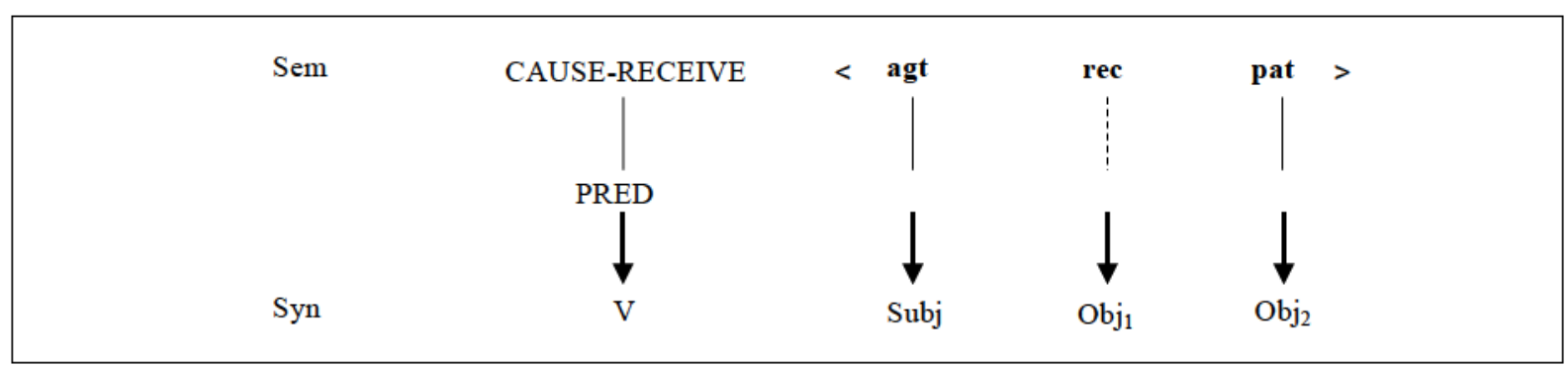

Abbildung 3: Vereinfachte Darstellung der Ditransitivkonstruktion nach Goldberg (1995, S. 50f. und S. 142).

Die kognitive Repräsentation grammatischen Wissens wird in der Konstruktionsgrammatik nicht als abgeschlossenes Modul betrachtet, das unabhängig von anderen kognitiven Mechanismen analysiert werden kann (Langacker 1987, S. 12-13; Lakoff 1987, S. 582; Croft 2001, S. 27; Tomasello 2003, Kap. 4; Goldberg 2006, Teil II). Vielmehr werden generelle, nicht sprachspezifische kognitive Mechanismen postuliert (Holismusannahme - Element der theoretischen Komponente). Die kognitive Repräsentation des sprachlichen Wissens besteht in der Konstruktionsgrammatik, wie das obige Beispiel zeigt, nicht in einer Menge rein syntaktischer Regeln, wie es in der Generativen Grammatik der Fall ist, sondern in einem System aus Konstruktionen bzw. Zeichen. Die produktiven Elemente - dies sind in der Generativen Grammatik die syntaktischen Regeln bzw. die parametrisierten Prinzipien und in der Konstruktionsgrammatik die schematischen Konstruktionen - werden im Gegensatz zur Generativen Grammatik in der Konstruktionsgrammatik nicht als von den lexikalischen Elementen (Generativen Grammatik) bzw. spezifischen Konstruktionen (Konstruktionsgrammatik) unterschieden angenommen. In der Konstruktionsgrammatik wird stattdessen ein Kontinuum von spezifischen Konstruktionen (z.B. Wörtern) zu schematischen Konstruktionen (produktive Einheiten) postuliert, da beides Form-Bedeutungs-Paare und somit prinzipiell gleiche Entitäten sind (Annahme des Lexikon-SyntaxKontinuums - Element der theoretischen Komponente).

Die schematischen Konstruktionen werden als durch Einsetzung spezifischerer Konstruktionen (z.B. Wörter) instanziiert angenommen. Diese Annahme nenne ich zur Abgrenzung von der Generativitätsannahme der Generativen Grammatik Deklarativitätsannahme (Element der theoretischen Komponente). Verschiedene Konstruktionstypen (z.B. Aktiv- und Passivkonstruktionen) besitzen in der Konstruktionsgrammatik jeweils eine eigene schematische Konstruktion. Somit werden im Gegensatz zur Generativen Grammatik keine Transformationsprozesse zwischen einer Tiefen- und einer Oberflächenstruktur angenommen, sondern es wird nur eine Repräsentationsebene behauptet (Monostratali-

\footnotetext{
${ }^{8}$ Goldberg (1995, S. 141).
} 
tätsannahme - Element der theoretischen Komponente). In einigen Arbeiten zur Generativen Grammatik werden beispielsweise Instanziierungen der oben vorgestellten Ditransitivkonstruktion aus tiefenstrukturellen Repräsentationen, die den Rezipienten repräsentierende Präpositionalphrasen enthalten, transformationell abgeleitet. So ist nach Larson (1988, S. 350-354) der Satz John sent Mary a letter aus der Tiefenstruktur John sent a letter to Mary abgeleitet, während in der Konstruktionsgrammatik beide Sätze als Instanziierungen verschiedener Konstruktionen angenommen werden (Goldberg 1995, S. 89-95).

Nach der Konstruktionsgrammatik werden die Elemente des Syntax-Lexikon-Kontinuums induktiv aus einzelnen Gebrauchsinstanzen generalisiert und sind somit aus dem Sprachgebrauch gewonnene, einzelsprachspezifische grammatische Repräsentationen (Goldberg 2006, Part II, 2009, S. 99-101; Bybee 2007). Die Existenz angeborenen grammatischen Wissens wird in der Konstruktionsgrammatik folglich explizit abgelehnt (Induktivismusannahme - Element der theoretischen Komponente). Eng verknüpft mit der Induktivismusannahme ist die Forderung der Gebrauchsbasiertheit (Element der methodologischen Komponente). Entsprechend dieser Forderung soll in der Konstruktionsgrammatik der Sprachgebrauch die Erklärungsgrundlage des Spracherwerbs und der kognitiven Repräsentation sprachlichen Wissens bilden. Somit steht die methodologische Forderung der Gebrauchsbasiertheit in einem Gegensatz zu wesentlichen methodologischen Forderungen und Unterscheidungen der Generativen Grammatik. Erstens spielt die Unterscheidung von Kompetenz und Performanz in der Konstruktionsgrammatik keine Rolle, da die kognitive Repräsentation sprachlichen Wissens aus dem Sprachgebrauch rekonstruiert wird: "[The] important methodological point is that the psycholinguistic units with which people operate are identified throughout observation of their language use" (Tomasello 2001, S. 62). Zweitens sind in der Konstruktionsgrammatik symbolische Einheiten (Konstruktionen) sowohl die Grundlage des Spracherwerbs als auch die Einheiten der kognitiven Repräsentation von Sprache. Hierdurch wird die in der Generativen Grammatik geltende grundlegende Unterscheidung von deskriptiver und explanativer Adäquatheit in der Konstruktionsgrammatik außer Kraft gesetzt. Drittens besitzt die in der Generativen Grammatik vorausgesetzte Unterscheidung von Kern und Peripherie in der gebrauchsbasierten Methodologie der Konstruktionsgrammatik keine Gültigkeit: Weder die spezifischen noch die Instanziierungen der schematischen Konstruktionen sind in irgendeiner besonderen Weise ausgezeichnet. Wie die Forderung der Gebrauchsbasiertheit, die Induktivismus- und die Holismusannahme nahelegen, ist die Konstruktionsgrammatik in der epistemologischen Tradition des Empirismus zu verorten, da die Annahme sprachspezifischen angeborenen Wissens abgelehnt wird und dieses stattdessen als durch generelle kognitive Mechanismen vollständig aus der Erfahrung erworben angenommen wird (empiristische Tradition - Element der methodologischen Komponente).

\subsection{Generative Grammatik und Konstruktionsgrammatik als koexistierende rivalisierende Paradigmen}

In der folgenden Tabelle werden die oben dargestellten Paradigmen der Generativen Grammatik und der Konstruktionsgrammatik bezüglich ihrer methodologischen, theoretischen, empirischen und programmatischen Komponenten gegenübergestellt, wobei ich die empirische und die programmatische Komponente zusammenfasse. Die programmatische Komponente besteht in der intendierten Ausweitung der innerhalb der Paradigmen durchgeführten Forschungsprogramme. Beispielsweise soll innerhalb der Generativen Grammatik die Erklärung systematischer grammatischer Unterschiede einiger Sprachen durch bestimmte Parameter auf die Unterschiede weiterer Sprachen ausgeweitet werden. Ein wichtiger Bestandteil der programmatischen Komponente der Konstruktionsgrammatik ist es, diejenigen Phänomene, die in der Generativen Grammatik dem Bereich der Kerngrammatiken zugeordnet sind, mittels des konstruktionsgrammatischen Instrumentariums erklären zu können.

\begin{tabular}{|c|c|}
\hline Generative Grammatik & Konstruktionsgrammatik \\
\hline \multicolumn{2}{|c|}{ Methodologische Komponenten } \\
\hline Performanz-Kompetenz-Dichotomie & \multirow{2}{*}{ Forderung der Gebrauchsbasiertheit } \\
\hline Deskriptive und explanative Adäquatheit & \\
\hline Kern-Peripherie-Dichotomie & Empiristische Tradition \\
\hline Cartesisch-rationalistische Tradition &
\end{tabular}




\begin{tabular}{|c|c|}
\hline \multicolumn{2}{|c|}{ Theoretische Komponenten } \\
\hline Generativitätsannahme & Deklarativitätsannahme \\
\hline Nativismusannahme & Induktivismusannahme \\
\hline Transformationsannahme & Monostratalitätsannahme \\
\hline Modularitätsannahme & Annahme des Syntax-Lexikon-Kontinuums \\
\hline Annahme der Syntax-Lexikon-Trennung & Spracherwerb \\
\hline \multicolumn{2}{|c|}{ Empirische und programmatische Komponenten } \\
\hline \multicolumn{2}{|c|}{ Sprachvergleich } \\
\hline \multicolumn{2}{|c|}{ Beschreibung und Klassifizierung grammatischer Phänomene } \\
\hline
\end{tabular}

Tabelle 1: Gegenüberstellung der methodologischen, theoretischen, empirischen und programmatischen Komponenten der Generativen Grammatik und der Konstruktionsgrammatik.

Tabelle 1 bietet erstens ein Argument dafür, dass es sich bei der Generativen Grammatik und der Konstruktionsgrammatik um Paradigmen im kuhnschen Sinn handelt, da die einzelnen Tabellenabschnitte die nach Kuhn konstitutiven Elemente für eine disziplinäre Matrix bzw. für ein Paradigma repräsentieren. Kuhns symbolische Verallgemeinerungen und Modelle können der theoretischen Komponente zugeordnet werden, Kuhns Werte entsprechen den methodologischen Voraussetzungen und Musterbeispiele im Sinne von Kuhn sind in der empirischen Komponente enthalten. Die Konstellation der Koexistenz von Generativer Grammatik und Konstruktionsgrammatik kann folglich nicht als eine vorparadigmatische bzw. vorwissenschaftliche Phase beschrieben werden (1976 [1962], Kap. II), da paradigmengeleitete Forschung existiert. Generative Grammatik und Konstruktionsgrammatik sind Paradigmen in einem kuhnschen Sinn. Die Linguistik ist somit - zumindest in den entsprechenden Unterdisziplinen - als eine reife Wissenschaft zu betrachten. Es ist folglich im Gegensatz zu Kuhns These der Vorwissenschaftlichkeit der Nicht-Naturwissenschaften inadäquat zu behaupten, dass lediglich die Naturwissenschaften reife Wissenschaften sind.

Zweitens zeigt sich in Tabelle 1, dass beide Paradigmen annähernd dieselben empirischen Gegenstandsbereiche zu erklären versuchen, allerdings auf der Grundlage gegensätzlicher methodologischer Voraussetzungen und theoretischer Annahmen bzw. Modellvorstellungen. ${ }^{9}$ Generative Grammatik und Konstruktionsgrammatik sind folglich unterschiedliche "Anbieter an einem gemeinsamen 'Markt"' (Schurz 1998, S. 4). Die beiden Paradigmen sind somit als koexistierende rivalisierende Paradigmen zu klassifizieren, zwischen denen aufgrund ihrer gegensätzlichen theoretischen Annahmen und Modellvorstellungen eine starke theoretische Divergenz besteht (vgl. Kornmesser und Schurz 2014b, S. 30-34). Kuhns These der Monoparadigmatizität der Wissenschaften ist somit empirisch falsifiziert.

\section{Multiparadigmatizität und Inkommensurabilität}

In der kuhnschen Konzeption einer zyklischen Abfolge von normaler Wissenschaft, wissenschaftlicher Revolution und einer darauf folgenden erneuten Phase der normalen Wissenschaft ist Inkommensurabilität ein Phänomen, das zwischen zwei Paradigmen auftritt, von denen das neuere Paradigma das ältere in der wissenschaftlichen Revolution abgelöst hat. Der frühe Kuhn bestimmt die Inkommensurabilität zweier Paradigmen als eine Art Wahrnehmungsverschiebung (Kuhn 1976 [1962], Kapitel X). Wissenschaftler inkommensurabler Paradigmen lebten in "verschiedenen Welten" und sähen Verschiedenes, wenn sie auf dasselbe schauten. Kuhn veranschaulicht das von ihm intendierte Verhältnis

\footnotetext{
${ }^{9}$ Ich spreche von annähernd denselben Gegenstandsbereichen und von gegensätzlichen theoretischen Annahmen anstatt von identischen Gegenstandsbereichen und widersprüchlichen theoretischen Annahmen, da die zwischen beiden Paradigmen bestehende semantische Inkommensurabilität erstens eine vollständige Übereinstimmung bezüglich der zu erklärenden empirischen Phänomene und zweitens im logischen Sinn widersprüchliche theoretische Annahmen verhindert (vgl. Abschnitt 4).
} 
inkommensurabler Paradigmen durch psychologische Gestaltwandelexperimente, bei denen z.B. ein und dasselbe Bild mal als Hase und mal als Ente gesehen wird. Man kann sagen, dass der frühe Kuhn Inkommensurabilität auf einer epistemologischen Ebene angesiedelt hat. Der späte Kuhn hingegen hat Inkommensurabilität aus einer eher sprachphilosophischen Perspektive betrachtet: Nach Kuhn (1983, 1993) besteht Inkommensurabilität in einer grundlegenden Begriffsverschiebung zwischen den Lexika der Sprachen zweier Paradigmen. ${ }^{10}$ Die in der Sprache eines Paradigmas $\mathrm{P}^{1}$ formulierten Aussagen lassen sich somit nicht in die Sprache eines Paradigmas $\mathrm{P}^{2}$ übersetzen. Die durch das Begriffsnetz des Paradigmas $\mathrm{P}^{1}$ repräsentierten Kategorien bilden mit den Extensionen der in $\mathrm{P}^{2}$ verwendeten Begriffe vielfache Überschneidungen, so dass eine Zuordnung bzw. Übersetzung nicht möglich ist. Eine Zuordnung bzw. Übersetzung der Begriffe durch eine Analyse der entsprechenden Definitionen ist ebenfalls ausgeschlossen, da nach Kuhns Begriffstheorie Definitionen höchstens eine untergeordnete Rolle als Intensionen von Begriffen spielen (vgl. Hoyningen-Huene 1989, Kapitel 3). Nach Kuhns Auffassung werden Kategorien statt durch Definitionen durch Ähnlichkeitsbeziehungen zwischen den Elementen einer Kategorie bestimmt. Der Wandel elementarer Ähnlichkeitsbeziehungen und die Etablierung neuer Ähnlichkeitsbeziehungen in einer wissenschaftlichen Revolution führen in der Folge zu einer Nicht-Übersetzbarkeit der beiden Begriffssysteme.

Dass zwischen den Paradigmen der Generativen Grammatik und der Konstruktionsgrammatik eine Inkommensurabilität im kuhnschen Sinne herrscht, werde ich im Folgenden an einer essentiellen Begriffsverschiebung zwischen den Lexika beider Paradigmen zeigen. Im Abschnitt drei wurde dargestellt, dass in der Generativen Grammatik sprachliche Entitäten entlang der grundlegenden Unterscheidung von Wörtern als Elementen des Lexikons einerseits und Instanziierungen von Regeln bzw. parametrisierten Prinzipien als Elementen der Syntax andererseits klassifiziert werden. Beide Kategorien - Lexikon und Syntax - besitzen keine Schnittmenge und entsprechen der Modellvorstellung zweier separater kognitiver Module, wie Abbildung 4 veranschaulicht:

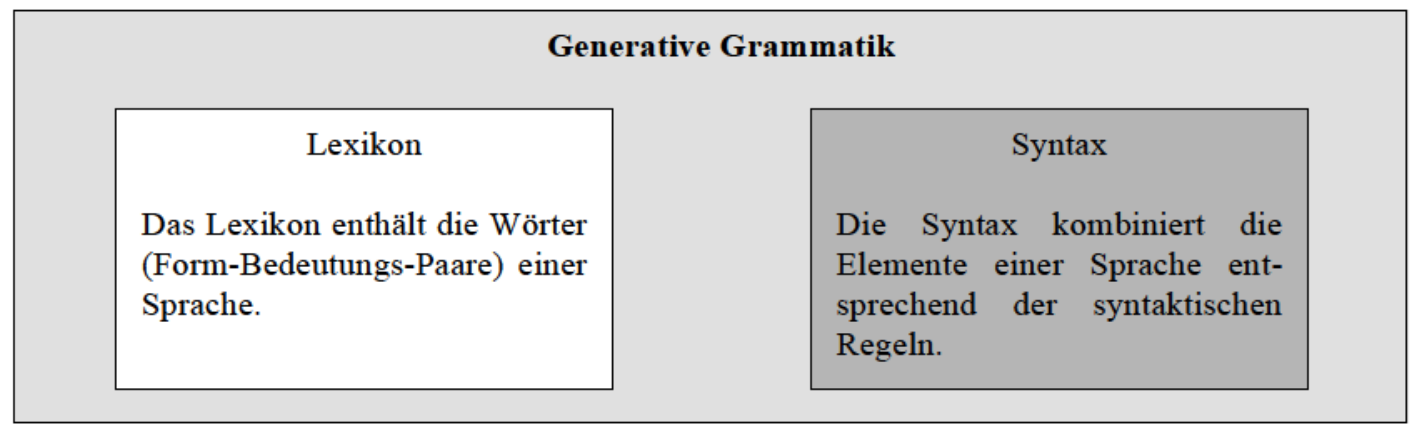

Abbildung 4: Schematische Darstellung der Syntax-Lexikon-Trennung der Generativen Grammatik.

Wie in Abschnitt drei gezeigt wurde, werden hingegen in der Konstruktionsgrammatik alle sprachlichen Entitäten als Konstruktionen bzw. als Instanziierungen von Konstruktionen und somit als prinzipiell gleiche Entitäten verstanden. Dies wird in der theoretischen Komponente durch die Modellvorstellung eines Kontinuums von Konstruktionen repräsentiert, wie es in Abbildung 5 dargestellt ist. Das Kontinuum reicht von spezifischen Konstruktionen wie Wörtern und Idiomen, die in der Generativen Grammatik dem Lexikon zugeordnet würden, zu schematischen Konstruktionen wie der oben vorgestellten Ditransitivkonstruktion. Da in der Konstruktionsgrammatik auch schematische Konstruktionen Form-Bedeutungs-Paare sind, können sie nicht mit den rein syntaktischen Regeln der Generativen Grammatik gleichgesetzt werden.

\footnotetext{
${ }^{10} \mathrm{Um}$ etwaigen Verwechslungen vorzubeugen, sei an dieser Stelle auf den Unterschied zwischen dem Lexikon als kognitiven Modul, das in der Generativen Grammatik angenommen wird, und dem Lexikon als Begriffsinventar eines Paradigmas, das Bestandteil einer wissenschaftlichen Sprache ist, hingewiesen.
} 


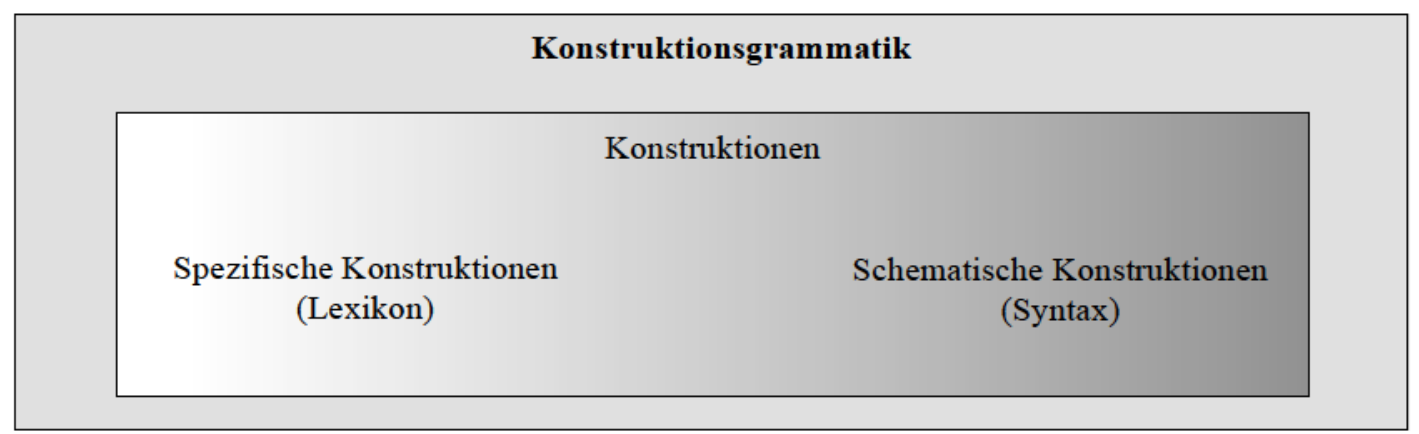

Abbildung 5: Schematische Darstellung des Syntax-Lexikon-Kontinuums der Konstruktionsgrammatik

Die durch die in beiden Paradigmen verwendeten grundlegenden Begriffe repräsentierte kategorielle Unterscheidung sprachlicher Entitäten entspricht Kuhns Vorstellung von Begriffsverschiebungen zwischen inkommensurablen Paradigmen. Wenn Wissenschaftler beider Paradigmen dieselben Sprachdaten vorliegen haben, dann "sieht" (in einem kuhnschen Sinne) der Generative Grammatiker Instanziierungen von Regeln bzw. parametrisierten Prinzipien, während der Konstruktionsgrammatiker Instanziierungen von Konstruktionen "sieht". Sie "sehen" unterschiedliche Entitäten entsprechend des von Kuhn (1976 [1962], Kapitel X und 2000 [1987], S. 15) angeführten Standardbeispiels von Ptolemäus und Kopernikus, die unterschiedliche Entitäten "sehen", wenn sie den Mond betrachten: Ptolemäus "sieht" auf der Grundlage seines Paradigmas einen Planeten und Kopernikus "sieht" einen natürlichen Satelliten.

Durch die Verschiebung der grundlegenden Klassifizierung sprachlicher Entitäten wird eine Übersetzung von Aussagen über Wörter und Regeln bzw. Prinzipien in Aussagen über Konstruktionen zwischen der Generativen Grammatik und der Konstruktionsgrammatik unmöglich. Es zeigt sich somit, dass die aus Kuhns zyklischem Verständnis der Wissenschaften und der These der Monoparadigmatizität der Wissenschaften folgende These der Nicht-Parallelität inkommensurabler Paradigmen nicht zutrifft. Die multiparadigmatische Struktur der Wissenschaften erzeugt parallele Normalwissenschaften von zueinander inkommensurablen Paradigmen.

In der auf Kuhn folgenden Diskussion über Inkommensurabilität zwischen Paradigmen, von denen das ältere in einer wissenschaftlichen Revolution durch das neuere ersetzt wird, ist es eine der zentralen Fragen, ob inkommensurable Paradigmen vergleichbar sind und ob es aufgrund der Inkommensurabilität wissenschaftlichen Fortschritt geben kann (vgl. Hoyningen-Huene und Sankey 2001). In der radikalen Interpretation der Inkommensurabilitätsthese wird diese Frage verneint. Kuhn (1983) selbst macht den Vorschlag, dass inkommensurable Paradigmen lediglich partiell inkommensurabel und folglich auch partiell kommensurabel sind. Dies steht allerdings in einem gewissen Widerspruch dazu, dass es sich bei den Begriffsverschiebungen um holistische Prozesse handelt (Kuhn 2000 [1987], S. 28), wonach folglich kein Ansatzpunkt für eine Kommensurabilität gegeben sein kann.

Das Pendant zu obiger Frage in Bezug auf koexistierende Paradigmen ist, ob bei inkommensurablen koexistierenden Paradigmen sinnvoll von einer Rivalität dieser Paradigmen gesprochen werden kann. Wie können zwei Paradigmen in einen Konflikt miteinander geraten, wenn es nicht möglich ist, die Aussagen beider Paradigmen ineinander zu übersetzen und anschließend einander gegenüberzustellen? Ich gehe nicht von der oben beschriebenen radikalen Interpretation der Inkommensurabilitätsthese aus, sondern versuche, den Konflikt zwischen Inkommensurabilität und Rivalität durch die Unterscheidung einer vorparadigmatischen und einer paradigmatischen Sprache zu beheben (vgl. Kornmesser 2014). Eine vorparadigmatische Sprache ist eine nicht von den koexistierenden Paradigmen abhängige Sprache, auf die Vertreter beider Paradigmen zurückgreifen können. Dies kann entweder eine von den Vertretern beider Paradigmen beherrschte Sprache eines vorgängigen Paradigmas oder eine geteilte natürliche Sprache sein. In Bezug auf das oben angeführte Standardbeispiel Kuhns bedeutet dies, dass sich Ptolemäus und Kopernikus über das weiße Objekt, das nachts am Himmel scheint, hätten unterhalten und in Bezug auf dieses Objekt Übereinstimmung in bestimmten Forschungsfragen erreichen können. Die Inkommensurabilität bezüglich dieses Objektes entsteht dann, wenn es mit den paradigmenspezifischen Sprachen erfasst wird, um es dem Theoriekern und den Theorieausprägungen der jeweiligen theoretischen Komponenten beider Paradigmen zuzuführen. Innerhalb des ptolemäischen 
Paradigmas wird dieses Objekt als Planet und innerhalb des kopernikanischen Paradigmas als natürlicher Satellit klassifiziert.

Anhand dieses zweistufigen Modells lässt sich analog zu dem kuhnschen Beispiel erklären, wie Generative Grammatiker und Konstruktionsgrammatiker trotz Inkommensurabilität über denselben Korpus von Sprachdaten sprechen und dieselben Forschungsfragen formulieren können. Erst wenn die entsprechenden Daten mittels der jeweiligen Paradigmen erfasst werden, um sie den jeweiligen theoretischen Komponenten zugänglich zu machen, entstehen die oben aufgezeigten Übersetzungsprobleme. Rivalität und Inkommensurabilität zwischen koexistierenden Paradigmen schließen sich somit nicht gegenseitig aus.

Nach Kuhns Vorstellung tritt Inkommensurabilität zwischen zwei Paradigmen $\mathrm{P}^{1}$ und $\mathrm{P}^{2}$ auf, wenn $\mathrm{P}^{2}$ das Paradigma $\mathrm{P}^{1}$ in einer wissenschaftlichen Revolution verdrängt hat. Die inkommensurablen Paradigmen werden somit nicht zeitgleich von wissenschaftlichen Gemeinschaften akzeptiert. Als Konsequenz hat die Inkommensurabilität keinen direkten Einfluss auf aktuelle wissenschaftliche Auseinandersetzungen, sondern ist eher von wissenschaftshistorischem Interesse. Dies zeigt sich unter anderem dadurch, dass dieses Phänomen von einem Wissenschaftshistoriker entdeckt wurde. In den hier betrachteten Fällen koexistierender Paradigmen ist die Problematik allerdings anders gelagert. Die inkommensurablen Paradigmen befinden sich gleichzeitig in einem wissenschaftlichen Diskurs, sofern sie sich nicht gegenseitig ignorieren. Dass und welchen Einfluss Inkommensurabilität auf koexistierende Paradigmen haben kann, wird u.a. im nächsten Abschnitt besprochen.

\section{Koexistierende Paradigmen und ihre faktischen Beziehungen}

Abschließend werde ich in diesem Abschnitt einen kurzen Überblick über die faktischen Beziehungen geben, die koexistierende Paradigmen in der wissenschaftlichen Praxis eingehen können (vgl. Kornmesser und Schurz 2014b, Abschnitt 5). Ich werde die Beziehungen der gegenseitigen Ignoranz, der destruktiven und konstruktiven Rivalität sowie der metaparadigmatischen Rivalität besprechen.

Die gegenseitige Ignoranz koexistierender Paradigmen besteht in der Nicht-Beachtung des jeweils anderen Paradigmas. Die Forschungsperspektive verweilt ausschließlich auf der normalen Wissenschaft des jeweils eigenen Paradigmas und divergierende Ansätze werden weder als (mögliche) Ergänzungen noch als kritische Hinterfragung der eigenen Position wahrgenommen. Vieles spricht dafür, dass die gegenseitige Ignoranz ein in der wissenschaftlichen Praxis häufig auftretendes Phänomen ist. Es ist allerdings problematisch, die Nicht-Beachtung von koexistierenden Paradigmen zu belegen. Beispielsweise kann nur aufgrund der Tatsache, dass in einzelnen Veröffentlichungen innerhalb der normalwissenschaftlichen Praxis eines Paradigmas rivalisierende Positionen nicht erwähnt werden, nicht unbedingt darauf geschlossen werden, dass die rivalisierenden Paradigmen ignoriert werden. Als Belege für die Ignoranz koexistierender Positionen können allerdings solche Beiträge angesehen werden, in denen innerhalb eines Paradigmas über die gesamte Disziplin gesprochen und dabei lediglich das eigene Paradigma betrachtet wird. Dies deutet auf eine Gleichsetzung der Disziplin mit dem eigenen Paradigma hin. Als ein Beispiel hierfür kann ein veröffentlichtes Interview mit Noam Chomsky angegeben werden, in dem Chomsky gebeten wird, die Entwicklung der Linguistik der letzten 20 Jahre zu bewerten (Chomsky 2004, S. 147-154). Dabei spricht er ausschließlich über die Entwicklung der Prinzipien- und Parametertheorie und des Minimalistischen Programms, die beide Bestandteil des Paradigmas der Generativen Grammatik sind, und blendet somit andere Entwicklungen der Linguistik aus.

In der destruktiven Rivalität wird das jeweils koexistierende Paradigma zwar als rivalisierende Position wahrgenommen, allerdings findet die Auseinandersetzung in der Regel auf einer rein ideologischen Ebene statt, die zu keinem fruchtbaren Ergebnis für eines der beiden Paradigmen führt. Als ein Beispiel für destruktive Rivalität führe ich die folgende Kritik am Paradigma der Konstruktionsgrammatik an, welche rein polemisch ist und inhaltlich an der Konstruktionsgrammatik vorbeigeht.

[Es] stellt sich die Frage nach dem Grund für die Attraktivität dieses Paradigmas [der Konstruktionsgrammatik]. Man macht es sich vermutlich zu leicht, wenn man Konstruktionsgrammatik als das Betätigungsfeld syntaktisch Unterbegabter bezeichnet, auch wenn dieser Vorwurf recht nahe liegt. [...]

[E]s werden auch die intelligenten Köpfe nicht mehr angezogen. Denn zur Erstellung von Konstruktionslexika braucht es viel Geduld und wenig Reflexion und In- 
novation. Schlimmer ist, dass der gesamte Erkenntniszuwachs der Linguistik im 20. Jahrhundert negiert wird. [...]

Bei konstruktionsgrammatischen Ansätzen handelt es sich nicht nur um ein unwissenschaftliches, sondern um ein antiwissenschaftliches Programm. (Leiss 2009, S. 19-27)

Die in dem Zitat gegebene Beschränkung der empirischen Komponente der Konstruktionsgrammatik auf die "Erstellung von Konstruktionslexika" ist keine adäquate Darstellung dieser Komponente, wie in Abschnitt 3.1 gezeigt wurde. Die Aussage, dass in der Konstruktionsgrammatik "der gesamte Erkenntniszuwachs der Linguistik im 20. Jahrhundert negiert" werde, ist eine Veranschaulichung einer typischen monoparadigmatischen Perspektive auf ein Konkurrenzparadigma, das durch eine unangemessene Darstellung bewertet wird.

In der konstruktiven Rivalität erzeugen koexistierende Paradigmen eine komplementäre Beziehung, die eine gegenseitige Ergänzung oder eine gegenseitige konstruktive Kritik bzw. einen Vergleich beider Paradigmen in Bezug auf eine bestimmte Forschungsfrage ermöglicht. Aufgrund der im vierten Abschnitt besprochenen Inkommensurabilitätsproblematik ist eine konstruktive Rivalität sehr voraussetzungsreich, da Wissenschaftler benötigt werden, die die paradigmatischen Sprachen beider Paradigmen beherrschen. Zudem muss das gemeinsame Forschungsobjekt in einer gemeinsamen vorparadigmatischen Sprache bestimmt werden. Für einen Vergleich oder eine gegenseitige Kritik müssen darüber hinaus metaparadigmatische Kriterien formuliert werden, um bewerten zu können, welches Paradigma in Bezug auf ein bestimmtes Forschungsproblem eine bessere Erklärung liefert (vgl. Kornmesser 2014). Ein Beispiel für einen Vergleich koexistierender Paradigmen bildet die Untersuchung deutscher Relativsätze sowohl auf einer generativistischen als auch auf einer konstruktionsgrammatischen Grundlage durch Webelhuth (2011). Die metaparadigmatischen Kriterien zur Bewertung der besseren Erklärung bilden hierbei die Einfachheit und die ontologische Sparsamkeit der Theorie.

Die metaparadigmatische Rivalität kann sich in denjenigen Disziplinen ausbilden, in denen die Paradigmenpluralität selbst zum Gegenstand der Betrachtung werden kann. Beispielsweise haben sich in der Soziologie, in der eine sehr ausgeprägte multiparadigmatische Struktur vorliegt, eine Reihe von soziologischen Ansätzen ausgebildet, die die koexistierenden soziologischen Paradigmen und deren Vergleich zum Gegenstand haben (vgl. Fischer 2014). Diese metaparadigmatischen soziologischen Positionen können wiederum als Paradigmen rekonstruiert werden, die dahingehend miteinander konkurrieren, dass sie den angemessensten Vergleich und die beste Erklärung für die Multiparadigmatizität der Soziologie bereitstellen. Eine wesentliche Herausforderung für die metaparadigmatischen Paradigmen besteht in der Überbrückung der im vierten Abschnitt besprochenen Inkommensurabilität zwischen den soziologischen Paradigmen, die den Gegenstand der Betrachtung bilden. Eine metaparadigmatische Rivalität dieser Art ist nur in solchen Disziplinen möglich, die prinzipiell in der Lage sind, eine Erklärung der multiparadigmatischen Struktur ihrer Disziplin zu geben. Die Soziologie kann beispielsweise soziologische Erklärungen der Multiparadigmatizität der Soziologie anbieten. Linguistische Erklärungen der Multiparadigmatizität der Linguistik sind hingegen nicht denkbar.

\section{Literatur:}

Ballmer, T. T. 1976. Inwiefern ist Linguistik empirisch? In Wissenschaftstheorie der Linguisitik, Hrsg. D. Wunderlich, 6-53. Kronberg: Athenäum Verlag.

Bloomfield, L. 1965 [1935]. Language. London: George Allen \& Unwin LTD.

Bybee, J. 2007. Frequency of Use and the Organization of Language. Oxford/New York: Oxford University Press.

Chomsky, N. 1971 [1957]. Syntactic Structures. Ninth Printing. Paris: Mouton.

Chomsky, N. 1959. Review of 'Verbal Behavior'. By B. F. Skinner. (The Century Psychology Series.) Pp. viii, 478. New York: Appleton-Century-Crofts, Inc., 1957. Language 35 (1): 26-58.

Chomsky, N. 1965. Aspects of the Theory of Syntax. Cambridge, Massachusetts: The MIT Press.

Chomsky, N. 1966. Cartesian Linguistics. New York/London: Harper\&Row.

Chomsky, N. 1975. Reflections on Language. New York: Pantheon Books.

Chomsky, N. 1972. Language and Mind. Enlarged Edition. New York/Chicago/San Francisco/Atlanta: Harcourt Brace Jovanovich.

Chomsky, N. 1980a. Rules and Representations. New York: Columbia University Press. 
Chomsky, N. 1980b. On Cognitive Structures and Their Development: A Reply to Piaget. In Language and Learning. The Debate between Jean Piaget and Noam Chomsky, Hrsg. M. Piatelli-Palmarini, 35-54. Cambridge, Massachusetts: Havard University Press.

Chomsky, N. 1981. Lectures on Government and Binding. Dordrecht: Foris Publications.

Chomsky, N. 1986. Knowledge of Language. New York: Praeger.

Chomsky, N. 1995. The Minimalist Program. Cambridge, Massachusetts: The MIT Press.

Chomsky, N. 2000. New Horizons in the Study of Language and Mind. Cambridge: Cambridge University Press. Chomsky, N. 2004. The Generative Enterprise Revisited. Berlin/New York: Mouton de Gruyter.

Chomsky, N. und J. Fodor. 1980. The Inductivist Fallacy. In Language and Learning. The Debate between Jean Piaget and Noam Chomsky, Hrsg. M. Piatelli-Palmarini, 255-275. Havard University Press: Cambridge, Massachusetts.

Cook, V. J. J. und M. Newson. 2007. Chomsky`s Universal Grammar. An Introduction. Third Edition. Malden: Blackwell Publishing.

Crain, S. und R. Thornton. 2006. Acquisition of Syntax and Semantics. In Handbook of Psycholinguistics, Hrsg. M. Traxler und M. A. Gernsbacher, 1073-1110. Amsterdam: Academic Press, Elsevier.

Croft, W. 2001. Radical Construction Grammar. Syntactic Theory in Typological Perspective. Oxford: Oxford University Press.

Croft, W. und D. A. Cruse. 2004. Cognitive Linguistics. Cambridge: Cambridge University Press.

Engelbert, S., A. Holler und K. Proost (Hrsg.). 2011. Sprachliches Wissen zwischen Lexikon und Grammatik. Institut für deutsche Sprache. Jahrbuch 2010. Berlin/Boston: de Gruyter.

Feyerabend, P. K. 1970. Consolations for the Specialist. In Criticism and the Growth of Knowledge, Hrsg. I. Lakatos und A. Musgrave, 197-230. Cambridge: Cambridge University Press.

Fillmore, C. J., P. Kay und M. C. O Connor. 1988. Regularity and idiomacity in grammatical constructions: The case of let alone. Language 64 (3): 501-538.

Fischer, J. 2014. Multiparadigmatizität der Soziologie. Übersichten, Unterscheidungen, Ursachen und Umgangsformen. In Die multiparadigmatische Struktur der Wissenschaften, Hrsg. S. Kornmesser und G. Schurz, 337-370. Wiesbaden: Springer VS.

Fischer, J. und M. Makroupolos (Hrsg.). 2004. Potsdamer Platz. Soziologische Theorien zu einem Ort der Moderne. München: Fink.

Gabriel, M, Gratzl, N. und D. Gruber. 2014. Zwischen akteurszentrierter und systemtheoretischer Soziologie. Eine Klassifikation der soziologischen Paradigmenstruktur. In Die multiparadigmatische Struktur der Wissenschaften, Hrsg. S. Kornmesser und G. Schurz, 305-335. Wiesbaden: Springer VS.

Goldberg, A. E. 1995. A Construction Grammar Approach to Argument Structure. Chicago/London: The University of Chicago Press.

Goldberg, A. E. 2006. Constructions at work. The nature of generalization in language. Oxford: Oxford University Press.

Hoyningen-Huene, P. 1989. Die Wissenschaftsphilosophie Thomas S. Kuhns. Brauschweig/Wiesbaden: Vieweg,

Hoyningen-Huene, P. und H. Sankey (Hrsg.). 2001. Incommensurability and Related Matters. Dordrecht/Boston/London: Kluwer Academic Publishers.

Kay, P. und C. Fillmore. 1999. Grammatical constructions and linguistic generalizations: the What's $X$ doing $Y$ ? construction. Language 75 (1): 1-33.

Kornmesser, S. 2014. Rivalisierende Paradigmen in der Linguistik. In Die multiparadigmatische Struktur der Wissenschaften, Hrsg. S. Kornmesser und G. Schurz, 229-270. Wiesbaden: Springer VS.

Kornmesser, S. und G. Schurz (Hrsg.). 2014a. Die multiparadigmatische Struktur der Wissenschaften. Wiesbaden: Springer VS.

Kornmesser, S. und G. Schurz. 2014b. Die multiparadigmatische Struktur der Wissenschaften: Einleitung und Übersicht. In Die multiparadigmatische Struktur der Wissenschaften, Hrsg. S. Kornmesser und G. Schurz, 11-46. Wiesbaden: Springer VS.

Kuhn, T. S. 1974 [1970]. Bemerkungen zu meinen Kritikern. In Kritik und Erkenntnisfortschritt, Hrsg. I. Lakatos, und A. Musgrave, 223-269. Vieweg: Braunschweig.

Kuhn, T. S. 1976 [1962]. Die Struktur wissenschaftlicher Revolutionen. Zweite Auflage. Frankfurt a. M.: Suhrkamp.

Kuhn, T. S. 1978 [1974]. Neue Überlegungen zum Begriff des Paradigma. In Thomas S. Kuhn. Die Entstehung des Neuen, Hrsg. L. Krüger, 389-420. Frankfurt a. M.: Suhrkamp.

Kuhn, T. S. 1983. Commensurability, Comparability, Communicability. In Proceedings of the 1982 Biennial Meeting of the Philosophy of Science Association. Volume 2, Hrsg. P. D. Asquith und T. Nickles, 669688. East Lansing: Philosophy of Science Association.

Kuhn, T. S. 1989. Possible Worlds in History of Science. In Possible Worlds in Humanities, Arts and Sciences, Hrsg. S. Allén, 9-32. Berlin [u.a.]: Walter de Gruyter.

Kuhn, T. S. 1993. Afterwords. In World Changes: Thomas Kuhn and the Nature of Science, Hrsg. P. Horwich, 311-341. Cambridge, Massachusetts: MIT Press. 
Kuhn, T. S. 2000 [1987]. What are Scientific Revolutions? In The Road since Structure. Hrsg. J. Conant und J. Haugeland, 13-32. Chicago \& London: The University of Chicago Press.

Lakatos, I. 1974 [1970]. Falsifikation und die Methodologie wissenschaftlicher Forschungsprogramme. In Kritik und Erkenntnisfortschritt, Hrsg. I. Lakatos und A. Musgrave, 89-189. Vieweg: Braunschweig.

Lakatos, I. und A. Musgrave (Hrsg.). 1974 [1970]. Kritik und Erkenntnisfortschritt. Vieweg: Braunschweig.

Lakoff, G. 1987. Women, Fire, and Dangerous Things. What Categories Reveal about the Mind. Chicago/London: The University of Chicago Press.

Langacker, R. 1987. Foundations of Cognitive Grammar. Volume I. Theoretical Prerequisites. Stanford (California): Stanford University Press.

Larson, R. K. 1988. On the Double Object Construction. Linguistic Inquiry 19: 335-391.

Laudan, L. 1977. Progress and its Problems. Berkeley [u.a.]: University of California Press.

Leiss, E. 2009. Konstruktionsgrammatik versus Universalgrammatik. In Wir sprechen und schreiben. Festschrift für Helmut Glück zum 60. Geburtstag, Hrsg. W. Eins und F. Schmöe, 17-28. Wiesbaden: Harrassowitz Verlag.

Lemke, M. und G. S. Schaal. 2014. Paradigmenpluralität in der Politikwissenschaft. Eine Bestandsaufnahme des Faches in Deutschland. In Die multiparadigmatische Struktur der Wissenschaften, Hrsg. S. Kornmesser und G. Schurz, 63-101. Wiesbaden: Springer VS.

Masterman, M. 1974 [1970]. Die Natur eines Paradigmas. In Kritik und Erkenntnisfortschritt, Hrsg. I. Lakatos, und A. Musgrave, 59-88. Braunschweig: Vieweg.

Müller, S. 1999. Deutsche Syntax deklarativ. Head-Driven Phrase Structure Grammar für das Deutsche. Tübingen: Niemeyer.

Müller, G. 2011. Regeln oder Konstruktionen? Von verblosen Direktiven zu sequenziellen Nominalreduplikationen. In Sprachliches Wissen zwischen Lexikon und Grammatik. Institut für deutsche Sprache, Hrsg. S. Engelbert, A. Holler und K. Proost, 211-249. Berlin/Boston: de Gruyter.

Münch, D. 1992. Einleitung: Computermodelle des Geistes. In Kognitionswissenschaft. Grundlagen, Probleme, Perspektiven, Hrsg. D. Münch, 7-53. Frankfurt a. M.: Suhrkamp.

Panaiotidi, E. 2014. Paradigmen in der Musikpädagogik. In Die multiparadigmatische Struktur der Wissenschaften, Hrsg. S. Kornmesser und G. Schurz, 145-179. Wiesbaden: Springer VS.

Pollard, C. und I. A. Sag. 1987. Information-Based Syntax and Semantics. Stanford: CSLI.

Pollard, C, und I. A. Sag. 1994. Head-Driven Phrase Structure Grammar. Chicago: University of Chicago Press.

Röhl, J. 2014. Feld oder Fernwirkung? Konkurrierende Paradigmen in der Elektrodynamik des 19. Jahrhunderts. In Die multiparadigmatische Struktur der Wissenschaften, Hrsg. S. Kornmesser und G. Schurz, 271-304. Wiesbaden: Springer VS.

Sankey, H. und P. Hoyningen-Huene. 2001. Introduction. In Incommensurability and Related Matters, Hrsg. Paul Hoyningen-Huene und Howard Sankey, vii-xxxiv. Dordrecht/Boston/London: Kluwer Academic Publishers.

Schurz, G. 1998. Koexistenzweisen rivalisierender Paradigmen. Eine begriffsklärende und problemtypologisierende Studie. In Koexistenz rivalisierender Paradigmen, Hrsg. G. Schurz und P. Weingartner, 1- 51. Oplanden/Wiesbaden: Westdeutscher Verlag.

Schurz, G. und P. Weingartner (Hrsg.). 1998. Koexistenz rivalisierender Paradigmen. Oplanden/Wiesbaden: Westdeutscher Verlag.

Taylor, J. R. 2002. Cognitive Grammar. Oxford: Oxford University Press.

Tomasello, M. 2001. First steps towards a usage-based theory of language acquisition. Cognitive Linguistics 11 : 61-82.

Tomasello, M. 2003. Constructing a Language. A Usage-Based Theory of Language Acquisition. Cambridge, Massachusetts/London: Havard University Press.

Webelhuth, G. 2011. Paradigmenwechsel rückwärts: Die Renaissance der grammatischen Konstruktion. In Sprachliches Wissen zwischen Lexikon und Grammatik. Institut für deutsche Sprache, Hrsg. S. Engelbert, A. Holler und K. Proost, 149-180. Berlin/Boston: de Gruyter.

Ziem, A. und A. Lasch. 2013. Konstruktionsgrammatik. Konzepte und Grundlagen gebrauchsbasierter Ansätze. Berlin/Boston: de Gruyter. 